\title{
Modelling the Propagation of Infectious Disease via Transportation Networks
}

Daniel J. Graham ( $\nabla$ d.j.graham@imperial.ac.uk )

Imperial College London

Anupriya -

Imperial College London

Prateek Bansal

National University of Singapore

\section{Research Article}

Keywords: COVID-19, infectious diseases, propagation, transportation networks, origin-destination, model

Posted Date: January 24th, 2022

DOI: https://doi.org/10.21203/rs.3.rs-1260315/v1

License: (c) (i) This work is licensed under a Creative Commons Attribution 4.0 International License.

Read Full License 


\title{
Modelling the propagation of infectious
}

\section{disease via transportation networks}

\author{
Anupriya $^{1 \dagger}$, Prateek Bansal ${ }^{2 \dagger}$ and Daniel J. Graham ${ }^{1 * \dagger}$ \\ ${ }^{1 *}$ Transport Strategy Centre, Department of Civil \& \\ Environmental Engineering, Imperial College London, London, \\ SW7 2AZ, UK. \\ ${ }^{2}$ Department of Civil and Environmental Engineering, National \\ University of Singapore, Singapore.
}

*Corresponding author(s). E-mail(s): d.j.graham@imperial.ac.uk;

Contributing authors: anupriya15@imperial.ac.uk; ceev236@nus.edu.sg;

${ }^{\dagger}$ These authors contributed equally to this work.

\begin{abstract}
The dynamics of human mobility have been known to play a critical role in the spread of infectious diseases like COVID-19. In this paper, we present a simple compact way to model the transmission of infectious disease through transportation networks using widely available aggregate mobility data in the form of a zone-level origin-destination (OD) travel flow matrix. A key feature of our model is that it not only captures the propagation of infection via direct connections between zones (that is, first-order effects) as in existing studies, but also transmission effects that are due to subsequent interactions in the remainder of the system (that is, higher-order effects). We demonstrate the importance of capturing higher-order effects in a simulation study. We then apply our model to study the first wave of COVID-19 infections in Italy using real-time data on mobility between Italian provinces (that is, provincelevel OD data) and daily reported caseloads at the same geographical level. Our empirical results indicate substantial predictive power, particularly during early stages of an outbreak. At least 85-percent of the spatial variation in observed weekly province-level COVID-19 cases can be forecasted using our proposed model. Further, from on our model,
\end{abstract}


we derive metrics that could help identify target areas for intervention, specifically during early outbreaks or resurgences of infectious diseases.

Keywords: COVID-19; infectious diseases; propagation; transportation networks; origin-destination; model

\section{Introduction}

COVID-19, the disease caused by Severe Acute Respiratory Syndrome Coronavirus-2 (SARS-CoV-2), has led to at least 269 million infections and claimed roughly 5.3 million lives in 221 countries and territories as of December 2021 [1]. Evidence suggests that similar to other respiratory viruses, SARSCoV-2 transmits between individuals via droplets, aerosols and fomites, either through direct contact or indirect contacts such as contaminated objects or surfaces [2]. Even after rapid and large-scale vaccinations, the disease has continued to spread worldwide, largely due to the emergence of new mutants of the virus and limited efficacy of the first-generation vaccines against reinfections from these variants [3]. Thus, non-pharmaceutical interventions based on social distancing, quarantine measures and other infection control practices continue to be of paramount importance to control the COVID-19 pandemic [4].

A key motivation behind such countermeasures is the need to limit human mobility because an increase in mobility is known to exacerbate the transmission of COVID-19 infections. Several previous studies have confirmed the positive association between mobility and propagation of pandemics and endemics in general, and COVID-19 in particular, offering insights at different spatial scales: continental, national, regional and local. For instance, [5-8] have explored the role of long distance travel in the spread of infectious diseases, while [9-14] have focused on dynamics of urban mobility and community 
transmission of infections. The propagation of infections has been analysed by exploiting various sources of mobility data, such as GPS data from mobile phones [10, 15-18], Google [14], and social media platforms such as Facebook [19-21] and Twitter [11]. There are two prominent strands of the literature on analysis of infection transmission using mobility data. The first strand adopts a detailed epidemiological model, such as the susceptible-exposed-infectiousremoved (SEIR) model, to simulate the importation of infections through mobility networks [see 22-24, for instance]. Such classic models, however, require various assumptions on population mixing, population compartment sizes and viral attributes such as incubation period [17]. The second strand of the literature leverages data on mobility patterns, mostly in real-time, to construct short-term forecasts of infection transmission risk [see 15-17, 20, 25, for instance]. In particular, such studies focus on the correlation between population flows, often represented in the form of aggregated origin-destination (OD) travel interactions between different locations in a given period, and the spatial distribution of infections in the subsequent period.

In this paper, we contribute to the second strand of the literature by reassessing and improving the utility of aggregated mobility data to model the potential propagation of infectious diseases such as COVID-19 through transportation networks. Specifically, we argue that a limitation of previous studies is that they have restricted analysis to the role of direct flows between the infected locations, say location $i$, and any other locations, say location $j$, in determining the diffusion of infections [see, for example, 20]. In addition to these direct or first-order effects, we emphasise that the propagation of infectious diseases can also occur through subsequent interactions in the transport system or higher-order effects. For instance, transmission first occurs because 
infection at location $i$ is delivered to locations $j$ via direct connections, but subsequently, infection propagates to locations $k$ through their interactions with $j$, and further to locations $l$ through their interactions with $k$, and so on. Accordingly, we reformulate the infection propagation process and propose an inverse connectivity matrix (ICM) to model this potential transmission of infections that captures both first- and higher-order connectivity effects. The resulting ICM is derived from an inverted origin-destination (OD) matrix. We further estimate a location-specific connectivity propagation metric (CPM) from the ICM, the value of which signifies the total risk of transmission of the infection in the system as a consequence of an additional infection in that location.

To show how our proposed matrix (that is, ICM) models the potential spread of COVID-19 through transportation networks, we simulate a synthetic OD matrix. The simulation demonstrates the importance of capturing higher-order connectivity effects in modelling propagation. We then evaluate the performance of our proposed matrix using province-level OD data and COVID-19 case load data from Italy. The OD data have been estimated by [18] from large-scale real-time mobility data of geo-located smartphone users in Italy. We use the mobility data to examine the correlation of our model forecasts with the observed province-level case data in future time periods. Our empirical results indicate substantial predictive power with more than 85 percent of the spatial variation in observed weekly province-level COVID-19 cases being predicted by our model.

We emphasise that similar to previous studies in this strand of the literature, we focus solely on predicting the transmission risk based on mobility patterns without precisely predicting future cases, which would require a disease transmission model. 
To the best of our knowledge, ours is the first analytical study to investigate the significance of higher-order network connectivity in the diffusion of infections through transportation networks and to encapsulate these effects in simple metrics. Our proposed approach could help policy makers identify high risk areas during early stages of disease outbreaks or target interventions for resurgences using readily available aggregate mobility data.

The remainder of this paper is organised as follows. Section 2 sets out the analytical framework we propose to model mobility and infections. Section 3 presents an empirical case study of COVID-19 propagation in Italy and reports results that assess the performance of our proposed method in forecasting transmission. Conclusions and recommendations are drawn in the final section.

\section{Modelling infection-mobility propagation via an inverse origin-destination (OD) matrix model}

We consider a typical OD matrix with the following layout:

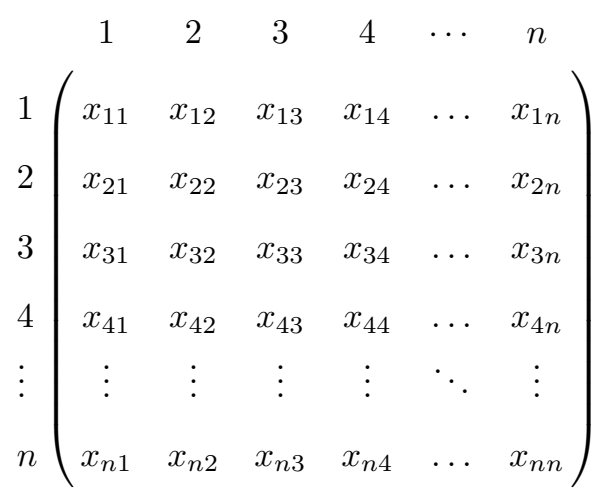

The cells of the OD matrix contain values $\left(x_{i j}\right)$ that measure transportation interactions between zones $i$ and $j$ in units of traffic volume such as trips. Reading across the rows of the matrix indicates trips that originate in zone $i$, 
$i=(1, \ldots, n)$, and end in $j,(j=(1, \ldots, n)$, while the columns show trips ending in $j$ from each zone $i$. For any origin $i$, the sum of the rows of the matrix gives total trips originating in $i$,

$$
O_{i}=\sum_{j=1}^{n} x_{i j},
$$

while for any destination $j$, the sum of the columns gives total trips ending in $j$,

$$
D_{j}=\sum_{i=1}^{n} x_{i j} .
$$

We multiply each element $x_{i j}$ of the OD matrix by the risk $r_{i}$ that an infected traveller from $i$ carries to destination $j$ to obtain the risk matrix $\boldsymbol{R}$ associated with the OD matrix. We adopt a heuristic approach to define $r_{i}$ based on qualitative information about epidemiological factors via active case counts. We construct $r_{i}$ using the number of active cases in zone $i$ normalised by the total number of active cases in all zones, that is,

$$
r_{i}=\frac{\text { Active cases in zone } i}{\sum_{i=1}^{n} \text { Active cases in zone } i} .
$$

Thus, the term $r_{i}$ represents the relative intensity of the disease in the origin zone $i$. We thus have the risk matrix $\boldsymbol{R}$ as follows:

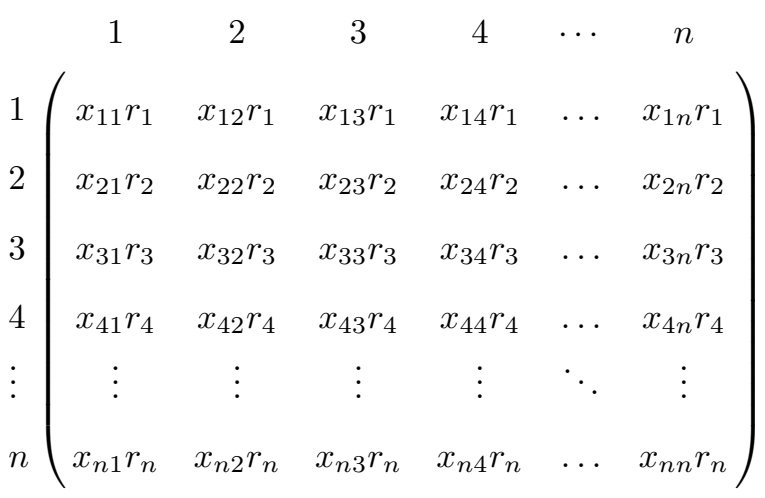


Each element $x_{i j} r_{i}$ of the matrix $\boldsymbol{R}$ gives the risk of infection in zone $j$ due to its first-order transportation interactions with zone $i$.

For any origin $i$, the sum of the rows of the matrix gives total risk of infection in all destinations due to trips originating in $i$,

$$
R_{O_{i}}=\sum_{j=1}^{n} x_{i j} r_{i}
$$

while the sum of the columns gives total risk of infection in zone $j$ due to trips ending in that zone,

$$
R_{D_{j}}=\sum_{i=1}^{n} x_{i j} r_{i} .
$$

The total risk of infection due to trips that either originate or end in zone $i$ is $R_{T_{i}}=R_{O_{i}}+R_{D_{i}}$, and total risk of infection due to trips made in the whole system, $T$, is

$$
R_{T}=\sum_{i=1}^{n} R_{O_{i}}=\sum_{j=1}^{n} R_{D_{j}} .
$$

We are interested in using the matrix to model potential propagation of infectious diseases like COVID-19 via transport networks. Given the presence of infection in the system, our assumption is that total risk of infection generated in a zone, say zone $i$, will be determined by:

1. Zonal activity scale - the scale of general interactions that occur between residents and non-residents active within the zone. For any zone $i$, the zonal activity scale $t_{i}$ can be proxied using the total risk of infection due to trips originating and ending in that zone as $t_{i}=r_{i} \times\left(O_{i}+D_{i}\right) / 2$. However, in most empirical applications, it can be shown that $t_{i} \approx r_{i} \times O_{i}=R_{O_{i}}$. We thus adopt the later definition. 
2. Zonal connectivity - the contagion that occurs via transport network connectivity. Crucially, for this component, we are concerned with both first-order and higher-order connectivity effects.

Accordingly, we write the total risk of infection generated in any zone $i$, or in other words, the total propagation potential of zone $i$ as

$$
p_{i}=t_{i}+\sum_{j=1}^{n} x_{j i} r_{j} .
$$

The first term on the right hand side of this equation represents the zonal activity scale. The second term, the sum of the column elements of the risk matrix, captures potential propagation via first-order connectivity to zone $i$ from all other zones $j$.

Following from equation 1, we propose a risk generation-propagation model shown in SI Appendix Table S.1. This table illustrates the dynamics of potential diffusion of infections in the system. One may note from this table that the total risk generated in any zone $i$ (that is, the sum of risk ended and zonal activity scale) equals the total risk propagated by that zone (that is, the sum of risk originated and risk imported). As we describe in the rest of this section, this table fulfils two separate functions. First, it is a descriptive framework for showing the relationship between risk of infection originating and ending in each zone and between inputs given by the risk originating from any zone and outputs given by the total risk propagated in the system. Second, it provides us with an analytical tool for measuring the impact of interventions on the total risk propagated in the system.

Notice that the transmission dynamics formulated in equation 1 do not appear to capture higher-order effects. To achieve this we re-formulate the 
model. We define $a_{i j}=x_{j i} r_{j} / p_{j}$ as a set of connectivity coefficients:

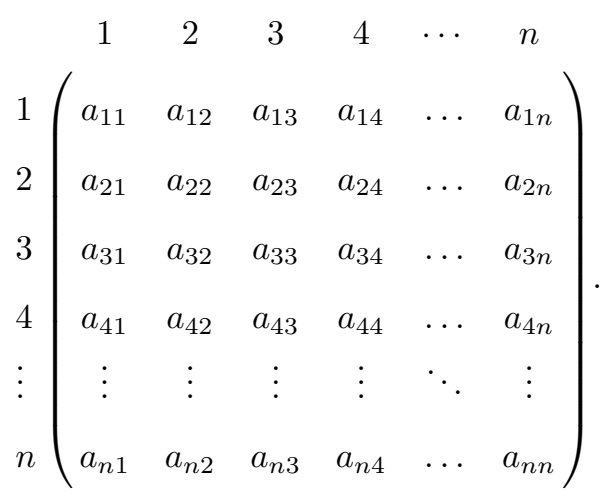

Note that the above matrix is essentially a transpose of the risk matrix $\boldsymbol{R}$, with every element $x_{j i} r_{j}$ normalised by their corresponding $p_{j}$. The cells of this matrix, $a_{i j}$, measure the risk of infection transmitted from zone $j$ to $i$ as a proportion of the total risk of infection propagated by zone $j$.

We can then write equation (1) above as:

$$
p_{i}=\sum_{j=1}^{n} a_{i j} p_{j}+t_{i} .
$$

Using matrix notation to represent the whole system, we have:

$$
\boldsymbol{p}=\boldsymbol{A p}+\boldsymbol{t},
$$

where $\boldsymbol{p}$ and $\boldsymbol{t}$ are column vectors of total zonal propagation potential and zonal scale respectively, and $\boldsymbol{A}$ is a $n \times n$ matrix of transportation interaction coefficients. Using the identity matrix $\boldsymbol{I}$, we can write

$$
(\boldsymbol{I}-\boldsymbol{A}) \boldsymbol{p}=\boldsymbol{t},
$$


allowing us to derive total potential system propagation via the following expression:

$$
\boldsymbol{p}=(\boldsymbol{I}-\boldsymbol{A})^{-1} \boldsymbol{t} .
$$

The inverse matrix $(\boldsymbol{I}-\boldsymbol{A})^{-1}$ is of key interest for our calculations. We refer to it as the inverse connectivity matrix (ICM). The cells of this matrix, say $b_{i j}$, measure successive infection effects from first and higher order interactions between zone $i$ and all other zones. Expanding the right hand side of (2) we can show how we capture higher-order effects in this re-formulation:

$$
(\boldsymbol{I}-\boldsymbol{A})^{-1} \boldsymbol{t}=\boldsymbol{t}+\boldsymbol{A} \boldsymbol{t}+\boldsymbol{A}^{2} \boldsymbol{t}+\boldsymbol{A}^{3} \boldsymbol{t}+\boldsymbol{A}^{4} \boldsymbol{t}+\cdots
$$

The first term in this expansion captures risk generated via zonal scale, the second term captures first-order propagation to any zone via transport network connectivity, the third term captures second-order connectivity propagation generated via the first order effects, term four is third-order effects generated by the second-order connectivity, and so on. In SI Appendix Section 2, we express the expansion terms in unit notation to illustrate the measure of higher-order effects. Further, in SI Appendix Section 3 attached, we present a simulation study that evaluates the role of higher-order network connectivity effects in the propagation of infections.

In addition to capturing transmission via both first and higher-order effects, we can use our model to calculate metrics that indicate the relative prevalence of infections in each zone of the system. For each zone $i$, we have $p_{i}$ that represents the total risk of infection generated in that zone. The resulting transmission of infections in the wider population in zone $i$ will depend on the susceptibility, $S_{i}$, of the population in zone $i$. We calculate zonal susceptibility, $S_{i}$, as the population of $i$ minus the cumulative cases in $i$, that is, 


$$
S_{i}=\text { Population of zone } i-\text { Cumulative cases in zone } i \text {. }
$$

Accordingly, we construct the infection prevalence metric $(\mathrm{P})$ as follows:

$$
\boldsymbol{P}=\boldsymbol{p} \odot \boldsymbol{S}
$$

where $\odot$ represents the Hadamard or element-wise product of the column vectors of zonal propagation potential $\boldsymbol{p}$ and zonal susceptibility $\boldsymbol{S}$. The P metric for each zone $i$ characterises the aggregate strength of mixing between the infected traveller from all zones $j$ and susceptible hosts in $i$. This metric can be matched with the observed raw case counts in the zone to assess the performance of the model.

Furthermore, note that the column sum of the ICM for each zone $i$, that is, $\sum_{j} b_{j i}$ measures the sum of risk propagated in the system, that is, to all zones $j, j=1,2, \ldots, n$, via both direct and indirect effects as a result of an additional infection in zone $i$. The resulting transmission of infections in the wider population will also depend on the susceptibility, $S_{j}$, of the population in zone $j$. Accordingly, we construct the required metric using equation 5 and refer to this metric as the connectivity propagation metric (CPM).

$$
C P M_{i}=\left(\sum_{j=1}^{n} b_{j i} * S_{j}\right)
$$

The higher the CPM metric for any zone, the higher is the potential number of infections in the system due to an infection in the zone and thus higher is the need for an intervention in that zone. The significance of the CPM estimates can be better understood using the unit notation representations attached in SI Appendix Section 2.

The CPM metric of zone $i$ can be multiplied by the number of active cases in the zone to estimate the net impact of all infections in zone $i$ on the system. 


\section{Case Study}

To gauge the predictive performance of the proposed matrix, we consider the first wave of COVID-19 infections in Italy. The earliest cluster of cases in Italy were detected in Lombardy and Veneto on February 21, 2020 and by the beginning of March 2020, the SARS-CoV-2 virus had spread to all Italian provinces. On March 21, 2020, a nation-wide lockdown was announced in Italy, which was gradually eased in May 2020. By June 3, 2020, freedom of movement across Italian regions and other European countries was restored.

Based on the above-discussed series of events, we consider the period between March 2020 to May 2020 as the study period. For this period, we investigate correlations between the observed weekly raw COVID-19 case counts in each province and the CPM of each province estimated using observed mobility patterns in the preceding week. A lag of one week between mobility patterns and raw case counts allows us to account for the transition time between exposure to case detection.

\subsection{Data}

We use a publicly available [26] mobility dataset comprising daily origindestination (OD) movements of population between Italian provinces for the period January 18, 2020 to June 26, 2020. These OD matrices were estimated by [18] for their work on assessing mobility changes in Italy in response to the COVID-19 lockdown. The matrices have been computed using a large-scale GPS data of about 170,000 de-identified smartphone users provided by Cuebiq Inc, a location intelligence and measurement platform.

Additionally, we obtain COVID-19 epidemiological data from a public repository [27] maintained by Il Dipartimento della Protezione Civile, which has also been used in previous studies such as [28]. The data comprises of a 
diurnal record of the cumulative numbers of positive COVID-19 cases in each Italian province. From these cumulative numbers, we calculate the daily numbers of new cases in each province. Note, however, that the data do not record the daily numbers of active cases. We presume the daily active case load in each zone to be a certain percentage the cumulative number of cases observed each day and further assume this percentage to not vary substantially across zones. Accordingly, we use the daily cumulative number of cases in each zone $i$ as a proxy for the daily number of active cases in that zone to estimate the risk $r_{i}$ and susceptibility $s_{i}$ of that zone, as defined in Section 2. Data on population size for each province is obtained from the official estimates provided by Il Istituto Nazionale di Statistica, ISTAT [29].

We use the risk and susceptibility estimates for a given week in conjugation with the corresponding aggregated OD matrix to generate the matrices $\boldsymbol{R}, \boldsymbol{A}$ and the inverse of $I-\boldsymbol{A}$, that is, the ICM. Thereafter, we produce provincelevel estimates of the prevalence metric $P$ and the connectivity propagation metric $C P M$ (see Section 2 for details). Correlations of the province-level $P$ estimates with raw infection counts in the subsequent week are then evaluated to assess the model performance. Finally, the province-level $C P M$ estimates are used to identify the top twenty provinces for any likely intervention.

\subsection{Results}

Before discussing our main results in Section 3.2.2, we present a brief precursor of our analysis in Section 3.2.1 where we assess the independent role of mobility data (that is, without epidemiological inputs) in determining the future spatial distribution of infections. 


\subsubsection{Preliminary analysis}

In general, the raw case incidences in any province $i$ in week $t$, say $I_{i, t}$, is a first-order auto-regressive process of the infections in that province. We input a network connectivity indicator given by $p_{i, t-1}$ in an auto-regressive model of infections in province $i$, given by equation 6 to understand its significance in the infection generation process. We derive $p_{i, t-1}$ from equation 1 using mobility data for week $t-1$, but ignoring any epidemiological inputs by setting the risk of each province as 1 , that is, $r_{j}=1 ; j=1, \ldots, n$. Essentially, $p_{i, t-1}$ sums the effect of transportation interactions between provinces $i$ and all provinces $j ; j=1, \ldots, n$ in week $t-1$ towards new infections generated in $i$, assuming all interactions to carry an identical risk of infection. We expect its effect on the dependent variable in equation 6 to be positive.

$$
I_{i, t}=\rho I_{i, t-1}+\beta p_{i, t-1}+\epsilon_{i, t}
$$

where, $\epsilon_{i, t}$ represents a idiosyncratic error term representing all random shocks to the dependent variable. The results from ordinary least squares estimation of equation 6 are presented in Table 1.

Table 1: Summary of results from the auto-regression infection generation model.

\begin{tabular}{lrr}
\hline Explanatory Variable & Coefficient & Std.Error \\
\hline Lag of raw case incidence (Dependent Variable) & $0.862^{* * *}$ & 0.013 \\
Network connectivity indicator $p$ & $276.864^{* * *}$ & 37.446 \\
Number of observations & & 1284 \\
\hline Note: Significance levels: $(* * *) 0.01,\left({ }^{* *}\right) 0.05,\left({ }^{*}\right) 0.1$ &
\end{tabular}

From Table 1, we note that the effect of the network connectivity covariate $p$ on observed raw case incidences is statistically significant at a 0.01 significance level. Further, as expected, the effect of the covariate is positive. 


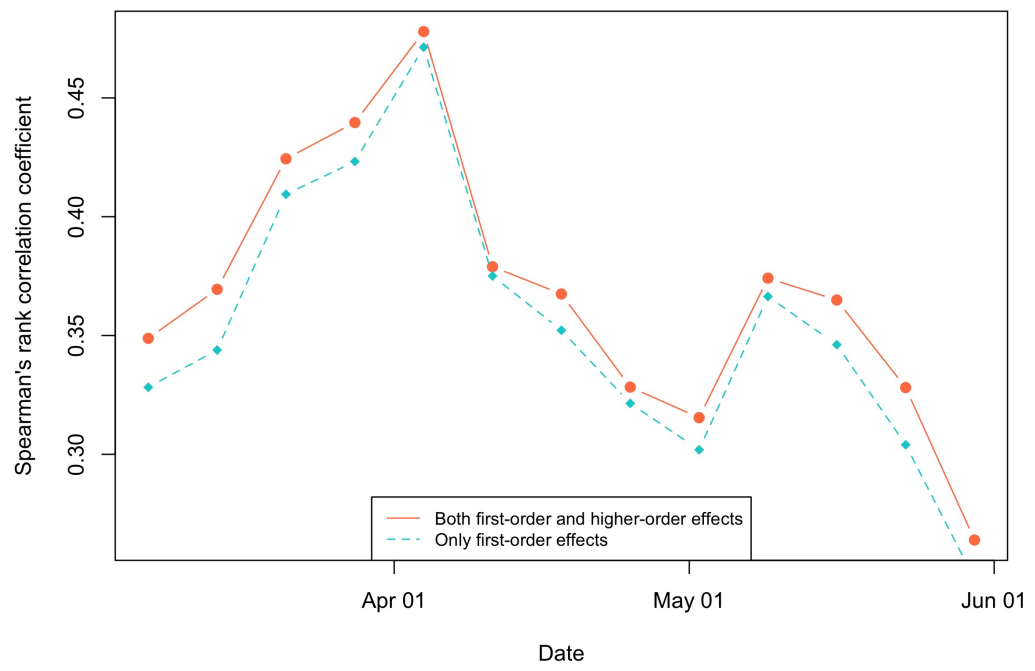

Fig. 1: Temporal variation of Spearman's rank correlation between raw case incidence and transmission risk derived from mobility data only.

We supplement this analysis by computing the Spearman's rank correlation coefficient $\left(r_{s}\right)$ between the province-level infection prevalence metric $P$ based on mobility data only (that is, setting the epidemiological inputs, risk $r_{i}$ and susceptibility $s_{i}$ to be 1 for all $\left.i=1, \ldots, n\right)$ and province-level observed case counts in the subsequent week. We use Spearman's correlation because we expect the relationship between the estimated transmission risk and the observed incidences in the subsequent week to be monotonic but not necessarily linear. Figure 1 shows temporal variation of $r_{s}$ over our study period. The figure indicates correlations between 0.35 to 0.48 for the first half of the wave of infections and between 0.26 to 0.38 for the latter half.

Similar to the results of [20], we observe in Figure 1 that there are diminishing marginal returns to the information added by mobility data as case counts grow. Specifically, we note from this figure that there is an optimal duration where the independent utility of mobility data is maximum. This optimal 
duration coincides with the peak of the infection wave when case counts are sufficiently high. Based on these results, we conclude that although mobility data has a substantial role to play in determining the relative distribution of infections in the system, its standalone use without inclusion of epidemiological factors as determinants of transmission risk will likely prove insufficient.

We also compute infection prevalence metric $P$ estimates by considering only first-order connectivity effects, that is, using $p \approx(\boldsymbol{I}+\boldsymbol{A}) \times t$ and inputting risk $r_{i}$ and susceptibility $s_{i}$ as 1 for all $i=1, \ldots, n$. Figure 1 plots the Spearman's rank correlation between these approximate $P$ values and observed cases in subsequent week. The figure shows that the prevalence metric based on all higher-order propagation effects outperforms that based on the first-order effects only, highlighting the significance of higher-order connectivity in the diffusion of infections through mobility networks.

\subsubsection{Main Results}

The province-level distributions of infection prevalence $P$ metrics for thirteen weeks, representing the relative distribution of risk of transmission of COVID19, are shown in Figure 3b and SI Appendix Figure S.3b to SI Appendix Figure S.14b. Additionally, Figure 3c and SI Appendix Figure S.3c to SI Appendix Figure S.14c illustrate the corresponding $P$ values computed using only firstorder connectivity effects, that is, computed using $(\boldsymbol{I}+\boldsymbol{A}) \times t$ and multiplying this sum with the corresponding zonal susceptibility. The spatial distribution of weekly case incidences across different provinces are shown in Figure 3a and SI Appendix Figure S.3a to SI Appendix Figure S.14a.

To validate the two different risk estimates, we compute the Spearman's rank correlation coefficient $\left(r_{s}\right)$ between these province-level $P$ estimates and province-level observed case counts in the subsequent week. Figure 2 shows temporal variation of $r_{s}$ over our study period. From this figure, we note that 
as we approach the peak of the first wave of COVID-19 infections in Italy, the value of $r_{s}$ increases from about 0.71 to 0.91 and then falls back to about 0.77 towards the end of this wave. We expect the actual correlations to be even higher as many COVID-19 case incidences might be missing non-randomly from the recorded data. Previous studies have suggested that rates of case ascertainment may vary substantially across locations depending upon the testing efforts by local officials. For instance, [30] estimated the ratio of confirmed to actual infections in Italy during the first wave to be only around $10 \%$. [28] suggested the use of hospitalisation counts as a better indicator or proxy for actual cases. However, province-level time-series data on hospitalisations in Italy is not available publicly.

Analogous to our observation from Figure 1, we find that our risk estimates generate better predictions of future case incidences during the peak of the infection wave when case counts in the entire system are quite high. This is because, as discussed in Section 3.2.1, the underlying contribution of mobility data is sizeable during this period. However, beyond this period, the local stringency and effectiveness of intervention measures driven by case counts may drive the distribution of raw case incidence [31]. Note that such unobserved epidemiological factors are proxied by $r_{i}$, which may primarily determine the correlations between risk estimates and case incidences in the latter half of the wave.

Figure 2 also indicates that the correlations of prevalence metric based on all higher-order propagation effects are mostly higher than those based on the first-order effects only, particularly during the first half of the wave when mobility data has the maximum utility in predicting future cases. This finding buttresses the significance of higher-order propagation effects. However, as discussed earlier, the extent of gains due to inclusion of higher-order effects 


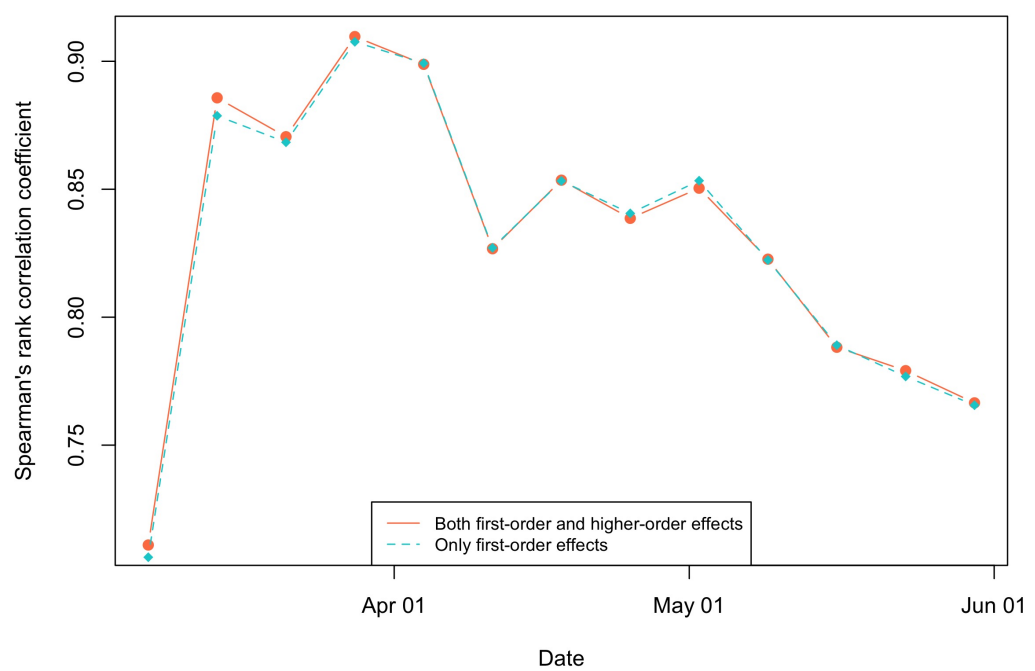

Fig. 2: Temporal variation of Spearman's rank correlation between case incidence and transmission risk derived from mobility data plus an indicator for epidemiological factors.

depends on the data generating process and can be more apparent in other case studies.

Finally, Figure 3d and SI Appendix Figure S.3d to SI Appendix Figure S.14d show the spatial distribution of the province-level $C P M$ values times their cumulative case counts (as a proxy for active caseload). As described in Section 2, for each province, this value signifies the transmission risk in the entire system due to the active infections in that province. Based on these figures, we identify the top twenty provinces in each period that should be prioritised for introduction of any likely intervention. We enlist these provinces in Table 3e and SI Appendix Table S.3e to SI Appendix Table S.14e. 


\section{Discussion}

This study presents a simple compact model of the potential transmission of infectious disease through transportation networks. It uses readily available aggregate mobility data along with corresponding data on disease incidences as a proxy for unobserved epidemiological factors to estimate the risk of transmission of infections in the following period. The model formulation provides three important quantities that encapsulate potential disease propagation dynamics in the entire network - the infection prevalence metric $(\mathrm{P})$, the inverse connectivity matrix (ICM) and the connectivity propagation metric (CPM). The $\mathrm{P}$ metric captures the time-varying spatial distribution of relative prevalence of the infectious disease in large geographical areas. The ICM is essentially derived from an inverted origin-destination travel flow matrix, and the cells of this matrix measure successive infection effects from first and higher order interactions between any zone $i$ and all other zones. Further, the sum of its columns is used to construct estimates of location-specific CPM. The CPM of a location quantifies the total risk of importation of the infectious disease in the entire system due to an infection in the location. The uniqueness of the proposed model stems from its ability to capture both first-order and higher-order interactions between different locations in the transportation network. We find that both first-order and higher-order connectivity effects are significant determinants of the potential diffusion of infections through mobility networks, but the relative importance of higher-order effects will likely vary by context.

We apply the proposed metrics to examine the first wave of COVID19 infections in Italy. The results show that our estimates of province-level COVID-19 transmission risk (that is, P) are highly correlated with the corresponding raw case counts in the subsequent period (Spearman's rank correlation coefficient between 0.71 and 0.91 ). Thus, our study reinforces the 
importance of aggregate mobility data to study the spread of infectious diseases and pandemics. Our results suggest that the mobility data yields the highest utility in terms of determining the spatial spread of infections at the early stages of outbreak or resurgence. Note that the role of our transmission risk estimates is limited to predicting the relative strength of outbreak across different geographical areas as opposed to predicting the actual case incidence.

The CPM estimates from this study could be particularly useful for public health authorities during the early stages of outbreak or resurgence of an infectious disease to identify target areas and the required magnitude of intervention. Moreover, the $\mathrm{P}$ estimates of an area could be employed as a performance metric to evaluate the effectiveness of intervention measures introduced in that area by benchmarking the risk predictions against real-time case counts.

\section{Declarations}

- Funding - This research did not receive any specific grant from funding agencies in the public, commercial, or not-for-profit sectors.

- Conflict of interest/Competing interests - The authors declare no competing interests.

- Availability of data and materials - This research uses data that is available in the public domain.

- Code availability - The authors are happy provide the code upon request.

- Author Contributions - A. conceptualised research, contributed methodology, analysed data, drafted the manuscript; P.B. conceptualised research, contributed methodology, edited the manuscript; D.J.G. conceptualised research, contributed methodology, edited the manuscript. 


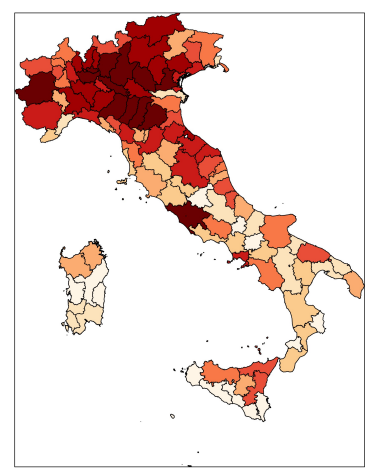

(a) A heatmap of observed cases.
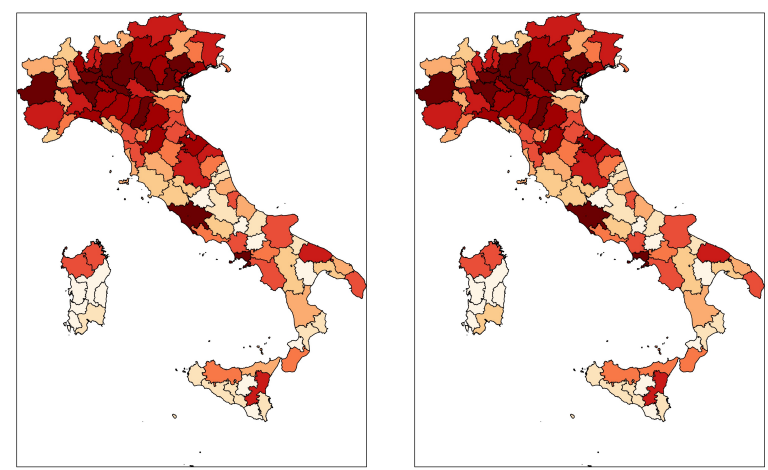

(b) A heatmap of trans- (c) A heatmap of transmission risk estimated via mission risk estimated via both first and higher con- first-order connectivity nectivity effects $\left[r_{s}=\right.$ effects $\left[r_{s}=0.9076\right]$. 0.9097].

\begin{tabular}{lc}
\hline $\begin{array}{l}\text { Name of the } \\
\text { province }\end{array}$ & $\begin{array}{c}\text { Rank (by CPM } \times \\
\text { active cases) }\end{array}$ \\
\hline Milano & 1 \\
Bergamo & 2 \\
Brescia & 3 \\
Roma & 4 \\
Torino & 5 \\
Napoli & 6 \\
Cremona & 7 \\
Padova & 8 \\
Monza e della Brianza & 9 \\
Verona & 10 \\
Pavia & 11 \\
Treviso & 12 \\
Lodi & 13 \\
Modena & 14 \\
Bologna & 15 \\
Venezia & 16 \\
Piacenza & 17 \\
Reggio Nell'Emilia & 18 \\
Vicenza & 19 \\
Genova & 20 \\
\hline
\end{tabular}

(d) A heatmap of estimated connectivity propagation metric times active case load. (e) List of top twenty provinces for likely intervention.

Fig. 3: Spatial distributions of estimated transmission risk and observed new COVID-19 cases in the week ending 28 March 2020. 


\section{References}

[1] WHO: https://covid19.who.int/. World Health Organisation (2021)

[2] Jayaweera, M., Perera, H., Gunawardana, B., Manatunge, J.: Transmission of COVID-19 virus by droplets and aerosols: A critical review on the unresolved dichotomy. Environmental research 188, 109819 (2020)

[3] Subbaraman, N.: How do vaccinated people spread Delta? What the science says. Nature 596(7872), 327-328 (2021)

[4] WHO: https://www.who.int/emergencies/diseases/ novel-coronavirus-2019/covid-19-vaccines/advice. World Health Organisation (2021)

[5] Grais, R.F., Ellis, J.H., Glass, G.E.: Assessing the impact of airline travel on the geographic spread of pandemic influenza. European journal of epidemiology 18(11), 1065-1072 (2003)

[6] Epstein, J.M., Goedecke, D.M., Yu, F., Morris, R.J., Wagener, D.K., Bobashev, G.V.: Controlling pandemic flu: the value of international air travel restrictions. PloS one 2(5), 401 (2007)

[7] Chinazzi, M., Davis, J.T., Ajelli, M., Gioannini, C., Litvinova, M., Merler, S., y Piontti, A.P., Mu, K., Rossi, L., Sun, K., et al.: The effect of travel restrictions on the spread of the 2019 novel coronavirus (COVID-19) outbreak. Science 368(6489), 395-400 (2020)

[8] Hu, M., Lin, H., Wang, J., Xu, C., Tatem, A.J., Meng, B., Zhang, X., Liu, Y., Wang, P., Wu, G., et al.: Risk of coronavirus disease 2019 transmission in train passengers: an epidemiological and modeling study. Clinical 
Infectious Diseases 72(4), 604-610 (2021)

[9] Wesolowski, A., Qureshi, T., Boni, M.F., Sundsøy, P.R., Johansson, M.A., Rasheed, S.B., Engø-Monsen, K., Buckee, C.O.: Impact of human mobility on the emergence of dengue epidemics in Pakistan. Proceedings of the National Academy of Sciences 112(38), 11887-11892 (2015)

[10] Ruktanonchai, N.W., DeLeenheer, P., Tatem, A.J., Alegana, V.A., Caughlin, T.T., zu Erbach-Schoenberg, E., Lourenço, C., Ruktanonchai, C.W., Smith, D.L.: Identifying malaria transmission foci for elimination using human mobility data. PLoS computational biology 12(4), 1004846 (2016)

[11] Huang, X., Li, Z., Jiang, Y., Li, X., Porter, D.: Twitter reveals human mobility dynamics during the COVID-19 pandemic. PloS one 15(11), $0241957(2020)$

[12] Xiong, C., Hu, S., Yang, M., Luo, W., Zhang, L.: Mobile device data reveal the dynamics in a positive relationship between human mobility and COVID-19 infections. Proceedings of the National Academy of Sciences 117(44), 27087-27089 (2020)

[13] Manout, O., Ciari, F.: Assessing the role of daily activities and mobility in the spread of COVID-19 in Montreal with an agent-based approach. Frontiers in Built Environment, 101 (2021)

[14] Yilmazkuday, H.: Stay-at-home works to fight against COVID-19: International evidence from Google mobility data. Journal of Human Behavior in the Social Environment 31(1-4), 210-220 (2021)

[15] Bengtsson, L., Gaudart, J., Lu, X., Moore, S., Wetter, E., Sallah, K., Rebaudet, S., Piarroux, R.: Using mobile phone data to predict the spatial 
spread of cholera. Scientific reports 5(1), 1-5 (2015)

[16] Iacus, S.M., Santamaria, C., Sermi, F., Spyratos, S., Tarchi, D., Vespe, M.: Human mobility and COVID-19 initial dynamics. Nonlinear Dynamics 101(3), 1901-1919 (2020)

[17] Jia, J.S., Lu, X., Yuan, Y., Xu, G., Jia, J., Christakis, N.A.: Population flow drives spatio-temporal distribution of COVID-19 in China. Nature 582(7812), 389-394 (2020)

[18] Pepe, E., Bajardi, P., Gauvin, L., Privitera, F., Lake, B., Cattuto, C., Tizzoni, M.: COVID-19 outbreak response: a first assessment of mobility changes in Italy following national lockdown. MedRxiv (2020)

[19] Bonaccorsi, G., Pierri, F., Cinelli, M., Flori, A., Galeazzi, A., Porcelli, F., Schmidt, A.L., Valensise, C.M., Scala, A., Quattrociocchi, W., et al.: Economic and social consequences of human mobility restrictions under COVID-19. Proceedings of the National Academy of Sciences 117(27), $15530-15535(2020)$

[20] Zachreson, C., Mitchell, L., Lydeamore, M.J., Rebuli, N., Tomko, M., Geard, N.: Risk mapping for COVID-19 outbreaks in Australia using mobility data. Journal of The Royal Society Interface 18(174), 20200657 (2021)

[21] Galeazzi, A., Cinelli, M., Bonaccorsi, G., Pierri, F., Schmidt, A.L., Scala, A., Pammolli, F., Quattrociocchi, W.: Human mobility in response to COVID-19 in France, Italy and UK. Scientific Reports 11(1), 1-10 (2021)

[22] Smieszek, T., Balmer, M., Hattendorf, J., Axhausen, K.W., Zinsstag, J., Scholz, R.W.: Reconstructing the 2003/2004 h3n2 influenza epidemic 
in switzerland with a spatially explicit, individual-based model. BMC infectious diseases 11(1), 1-18 (2011)

[23] Chang, S., Pierson, E., Koh, P.W., Gerardin, J., Redbird, B., Grusky, D., Leskovec, J.: Mobility network models of COVID-19 explain inequities and inform reopening. Nature 589(7840), 82-87 (2021)

[24] Wu, J.T., Leung, K., Bushman, M., Kishore, N., Niehus, R., de Salazar, P.M., Cowling, B.J., Lipsitch, M., Leung, G.M.: Estimating clinical severity of COVID-19 from the transmission dynamics in Wuhan, China. Nature medicine 26(4), 506-510 (2020)

[25] Badr, H.S., Du, H., Marshall, M., Dong, E., Squire, M.M., Gardner, L.M.: Association between mobility patterns and COVID-19 transmission in the USA: a mathematical modelling study. The Lancet Infectious Diseases $\mathbf{2 0}(11), 1247-1254(2020)$

[26] HDX: https://data.humdata.org/dataset/covid-19-mobility-italy. The Humanitarian Data Exchange (2021)

[27] Italy: https://github.com/pcm-dpc/COVID-19. Il Dipartimento della Protezione Civile (2021)

[28] Bertuzzo, E., Mari, L., Pasetto, D., Miccoli, S., Casagrandi, R., Gatto, M., Rinaldo, A.: The geography of COVID-19 spread in Italy and implications for the relaxation of confinement measures. Nature communications 11(1), $1-11(2020)$

[29] Italy: http://citypopulation.de/en/italy/admin/. Il Istituto Nazionale di Statistica (2021) 
[30] Gatto, M., Bertuzzo, E., Mari, L., Miccoli, S., Carraro, L., Casagrandi, R., Rinaldo, A.: Spread and dynamics of the COVID-19 epidemic in Italy: Effects of emergency containment measures. Proceedings of the National Academy of Sciences 117(19), 10484-10491 (2020)

[31] Sen, S., Karaca-Mandic, P., Georgiou, A.: Association of stay-at-home orders with COVID-19 hospitalizations in 4 states. Jama 323(24), 2522$2524(2020)$ 


\section{Supplementary Files}

This is a list of supplementary files associated with this preprint. Click to download.

- Appendix.pdf 